\title{
Politique
}

\section{La démocratie du secret : le contrôle des activités de renseignement au Canada}

\section{Janine Krieber}

Numéro 13, printemps 1988

Crise de décision

URI : https://id.erudit.org/iderudit/040583ar

DOI : https://doi.org/10.7202/040583ar

Aller au sommaire du numéro

Éditeur(s)

Société québécoise de science politique

ISSN

0711-608X (imprimé)

1918-6584 (numérique)

Découvrir la revue

Citer cet article

Krieber, J. (1988). La démocratie du secret : le contrôle des activités de renseignement au Canada. Politique, (13), 37-62.

https://doi.org/10.7202/040583ar d'utilisation que vous pouvez consulter en ligne.

https://apropos.erudit.org/fr/usagers/politique-dutilisation/ 


\title{
LA DÉMOCRATIE DU SECRET: LE CONTRÔLE DES ACTIVITÉS DE RENSEIGNEMENT AU CANADA
}

\author{
Janine Krieber \\ Université Laval
}

L'information, c'est bien connu, est une clef de la puissance. "Connais ton ennemi", un des premiers préceptes de toute bonne stratégie. Les hommes qui exercent le pouvoir sont donc insatiables de renseignements, autant sur l'ennemi extérieur que sur celui qui se terre à l'intérieur des frontières. Le droit de tout savoir sur tous semble presque, au désespoir des défenseurs des libertés, faire partie de la légitimité qui fonde l'État moderne. Un service de renseignement bien organisé et efficace est, de tous les services gouvernementaux, la perle, l'enfant chéri du politique, en même temps qu'un repoussoir, le bâtard qu'on est prompt à renier.

En démocratie parlementaire, la relation du politique au renseignement est ambiguë. Parce que l'activité secrète reste un espace nécessairement soustrait à la «transparence démocratique ", elle est, par nature, inacceptable. Parce qu'instrument de protection de la souveraineté, du régime et des institutions, elle est indispensable à la survie de cette démocratie dont elle est la négation. Beau dilemme.

En 1981, dans le plus pur style "Commission», le rapport McDonald posait ainsi le problème:

Politique, 13 (Printemps 1988). 
«... il s'agit de protéger la démocratie contre ses ennemis de l'intérieur comme de l'extérieur, sans la détruire pour autant.

[...] L'État démocratique libéral est le seul dont on s'attend qu'il évite que les enquêtes menées sur les activités subversives ne portent atteinte aux libertés de dissidence politique et d'association, fondements d'une société libre. "

Tout un programme. Nous verrons, dans les pages qui suivent, quelles sont les difficultés d'application de cette volonté de démocratisation des activités de renseignements au Canada. Les contraintes tiennent à une contradiction irréconciliable entre le secret d'État et la démocratie, comprise comme sanction populaire, régulière et obligatoire, de l'activité politique; dépendante donc des libertés d'expression et surtout d'information.

Ces dernières années, le gouvernement canadien a procédé à un réaménagement dans la communauté du renseignement. Redéfinitions législatives, organisationnelles et, de façon plus profonde, une tentative de transformation des mentalités dans les services spécialisés. Beaucoup a été fait pour répondre aux critiques, principalement en ce qui concerne les activités anti-subversives. Le 25 février 1988, le Solliciteur général annonçait que le Service de renseignement fermait $95 \%$ de ses dossiers sur les «éléments subversifs ${ }^{2}$. Un peu plus tôt, à la suite du rapport Osbaldeston, on apprenait la dissolution de la direction anti-subversion maintenant fondue dans la direction anti-terrorisme ${ }^{3}$.

Que ces réformes aient été possibles montre que, dans le monde du renseignement, les significations ne sont pas adoptées

1. Commission d'enquête sur certaines activités de la Gendarmerie royale du Canada, La liberté et la sécurité, Deuxième rapport - Volume 1. Centre d'édition du gouvernement du Canada, Ottawa, 1981, pp. 43-44.

2. Solliciteur général du Canada, Communiqué, 25 février 1988.

3. "Il y a lieu d'éliminer la Direction de l'anti-subversion et de réaffecter ses fonctions. Les activités influencées par l'étranger et préjudiciables aux intérêts du Canada devraient relever de la fonction contre-espionnage, et les actes de violence grave ayant pour but la réalisation d'objectifs politiques, de la fonction anti-terrorisme. " Gordon F. Osbaldeston, Des ressources humaines et un processus en transition: Rapport du Groupe consultatif indépendant au Solliciteur général sur le Service canadien du renseignement de sécurité. Ministre des Approvisionnements et services Canada, 1987, recommandation 15, p. 39. 
une fois pour toutes et fluctuent au rythme de certaines volontés politiques. Mais le projet, maintes fois exprimé, de démocratisation du renseignement se heurte à de fortes contraintes du milieu. Contraintes de structure et de culture. Les premières sont liées aux caractéristiques intrinsèques de la communauté du renseignement: éclatement du système dans le cloisonnement des services et centralisation de l'information. D'où une difficulté permanente de rendre compte de la totalité, puisque toute information même à l'intérieur semble-t-il — reste partielle. D'autres problèmes viennent de la nature même de l'activité de renseignement. La mission est de garantir la sécurité des citoyens sur une base de dissuasion et de prévention. Son succès maximal serait donc qu'aucune menace pèse sur qui que ce soit; du même souffle, le service perd sa raison d'être. Contradiction difficile à dépasser. Moins il y a de cibles, plus le service s'avère efficace et moins il est nécessaire.

Dans le travail quotidien, ces contraintes structurelles trouvent leur dépassement partiel dans l'élaboration d'une culture organisationnelle spécifique au renseignement. Cette culture est fondée sur la nature à dominance policière de l'organisation et sur ses privilèges en tant que dépositaire du secret d'État. L'organisation est garante de la survie de l'État en assurant sa sécurité. Vaste et importante mission qui confere aux individus qui y participent un statut particulier qui se traduit par une solidarité à toute épreuve devant la critique.

Ces caractéristiques donnent à l'organisation de renseignement une large marge de manœuvre quant à l'évaluation de la menace. Une relative liberté d'action et de définition, renforcée par les imprécisions législatives, provoque une sur-évaluation des cibles potentielles et c'est un service du gouvernement lui-même qui devient une menace pour les libertés démocratiques. Nous verrons comment, à travers les différentes enquêtes qui ont évalué les services secrets canadiens, se sont posés les problèmes de restructuration, quelles solutions ont été proposées et adoptées; enfin, 
de quelle nature peuvent être les résistances au changement et dans quelle mesure elles peuvent dénaturer les efforts de réforme.

\section{La communauté du renseignement}

Un peu partout, dans les ministères fédéraux, dans les différents corps de police, des services collaborent aux activités de renseignement ${ }^{4}$. Plusieurs organismes qui n'ont pas a priori de mission de renseignement spécifique contribuent à amasser les informations qui, une fois traitées par les services spécialisés, deviendront du «renseignement». Les sources sur l'histoire et l'organisation des activités secrètes du gouvernement canadien sont peu nombreuses et les fonctionnaires peu loquaces. Le chercheur doit donc accorder un minimum de crédibilité aux rares informations qui filtrent du gouvernement lui-même sous forme de rapports. Pour ce qui est de la communauté du renseignement, on peut estimer que, mis à part la création du SCRS, peu de choses ont changé depuis la publication du rapport McDonald. Le travail de la communauté du renseignement s'articule, en gros, à trois niveaux techniques: 1) l'information, de sources ouvertes (médias et fichiers gouvernementaux) ou d'opérations secrètes; 2) l'analyse par les spécialistes de sujets ou de régions; 3) l'élaboration de plans de crise qui, idéalement, iraient de l'alerte à la bombe jusqu'à la guerre nucléaire.

Au ministère des Affaires extérieures, la Direction de la sécurité a charge de protéger les personnes et les biens, surtout les diplomates en poste, et la Section de la sécurité nationale s'occupe des diplomates étrangers au Canada. Ces services ont besoin d'évaluations précises sur les risques encourus presque partout

4. On trouvera dans le rapport de la Commision McDonald op. cit. un très bon chapitre qui résume en gros la littérature sur l'histoire du renseignement au Canada: «Le dispositif mis en place par le gouvernement» p. 49-95. 
au monde. C'est la Direction de l'analyse des renseignements qui doit tenir un recueil de données sur les divers coins du globe et coordonner les mesures d'urgence. En plus de ces évaluations politiques, les Affaires extérieures se chargent du renseignement pour les ministères à vocation économique. Dans la communauté du renseignement, et ceci est vrai pour tous les pays, c'est le réseau presque mondial des ambassades qui fait la qualité et l'efficacité de l'organisation. Le flux continu d'informations, lorsque bien traité, peut donner aux analystes l'outil essentiel d'une bonne évaluation de risque, tant domestique qu'outre-frontière.

Le deuxième pilier de la cueillette d'informations s'appuie sur la Commission de l'emploi et de l'immigration du Canada et Revenu Canada (douanes et accises) qui doivent fournir des renseignements sur certaines catégories de visiteurs "à risque» qui pourraient être terroristes, agents de renseignements, membres d'organisations «subversives» ou de «façade». Les Affaires extérieures donnent une image des mouvements politiques et économiques internationaux, la Commission fournit des informations sur les personnes. Pour un service de renseignement, ses dossiers sont le centre du filtrage de sécurité.

Sur ces bases, travaillent un certain nombre de services spécialisés ou sectoriels. Le ministère de la Défense nationale a un Service des enquêtes spéciales qui ne peut se servir de moyens secrets mais a un Programme de liaison police et sécurité. Le ministère des Approvisionnements et services doit assurer la sécurité des industries et du matériel informatique, préparer l'industrie à toute éventualité et voir à l'approvisionnement en temps de guerre ou de crise. Finalement, le Centre de la sécurité et des télécommunications, intégré au ministère de la Défense, surveille le secret des communications gouvernementales. 
Ceci n'est que l'essentiel du système; seulement dresser une liste des organismes est une entreprise en $\operatorname{soi}^{5}$. Un rapport du Sénat sur le terrorisme et la sécurité publique a dénombré près de 20 agences et départements fédéraux impliqués seulement dans la lutte anti-terroriste, que ce soit dans l'action sur le terrain, la prévention ou les coordinations politique et administrative. Le rapport se demande même si tout ceci peut véritablement fonctionner ${ }^{6}$.

Le renseignement c'est donc plus que l'idée première qu'on se fait de l'espionnage ${ }^{7}$. Tout un réseau travaille à rassembler l'information et à la traiter. Le matériel est construit en grande partie, dans la mesure où on peut en juger de l'extérieur, à partir de sources ouvertes. Cette partie de l'activité de renseignement pose des problèmes éthiques différents de ceux suscités par les opérations secrètes. Le recours aux sources ouvertes soulève la question du droit de l'administration à rassembler les informations dont elle dispose concernant la vie privée des citoyens et des

5. Ce fut une des premières tâches du CSARS: «En fait, lorsque nous avons assumé nos fonctions en 1984, nous avons été surpris de ne pas même pouvoir trouver un annuaire complet des organismes du gouvernement fédéral travaillant dans le secteur de la sécurité et du renseignement.[...] Incapables de trouver un annuaire complet, nous avons décidé d'en faire un nous-même, tâche que nous avons terminée en 1986-1987. Nous reconnaissons avec regret, que nous ne pouvons pas rendre cet annuaire public. Il contient des informations - notamment des descriptions des rôles joués par certaines agences - qui seraient beaucoup trop intéressantes pour les ennemis de notre pays." Rapport 1986-1987, p. 78.

6. William M. Kelly, The Report of the Senate Special Commitee on Terrorism and the Public Safety, Ottawa, Minister of Supply and Services Canada, 1987, part II: "The Federal Government's Counter and Anti-Terrorism Establishment ", p. 45-70. - Une version française devrait obligatoirement exister mais reste, pour quelque raison, inaccessible.

7. On comprendra aisément que ce genre d'activités se prête peu aux représentations administratives classiques de type "organigramme». Le réseau du renseignement est quelque chose de formel/informel qui s'organise autour de différents domaines spécialisés d'intervention et d'intérêt. C'est pourquoi il est constamment en mouvement et suit les définitions ponctuelles des problèmes abordés. Le dernier exemple de transformation est la refonte, en un seul, des services anti-subversion et anti-terrorisme (symptôme de nouvelles définitions administratives de la subversion et du terrorisme). Pour un organigramme concernant les interventions anti-terroristes: William M. Kelly, op. cit, p. 51. 
visiteurs. Le problème posé par les opérations clandestines, que ce soit la simple écoute téléphonique jusqu'à-l'infiltration d'associations légales de protestation, vient du fait que l'administration initie une action contre des citoyens, à leur insu, et on verra plus loin que les balises qui dictent la suspicion sont loin d'être claires.

Les gouvernements canadiens ont toujours eu beaucoup de réticences à admettre que certains services pouvaient se livrer à des activités secrètes. Bien qu'existant probablement depuis les années 1860, ce n'est qu'en 1934 qu'un gouvernement a admis officiellement l'existence d'un service secret au sein de l'administration ${ }^{8}$. L'organisation et le contrôle de ces activités sont restés une préoccupation récurrente quoique marginale pour les gouvernements; les activités d'espionnage et de contre-espionnage étant étroitement liées à la politique étrangère. C'est au cours des années 1960, au moment de la cristallisation des contestations autour de la «Nouvelle gauche», de l'opposition à la guerre du Vietnam aux États-Unis et de l'émergence du nationalisme violent au Québec, que se sont intensifiées les activités "anti-subversives» du renseignement et que les cibles sont par conséquent devenues intérieures.

En 1966 a été formée la Commission royale d'enquête sur la sécurité, dite Mackenzie. Le rapport déposé en 1968 proposait trois transformations importantes: 1) La création d'un Secrétariat à la sécurité, responsable de la formulation des politiques; 2) le détachement des services de sécurité de la GRC et leur transformation en organisme civil; 3) la mise sur pied d'un Comité de révision?' Ces recommandations n'ont été suivies que de quelques réformes jusqu'en 1977, date de la nomination du commissaire Simmonds.

8. Commission d'enquête sur certaines activités de la Gendarmerie royale du Canada. Op. cit, p. 60: «... ce n'est qu'en 1934 qu'un ministre de la justice a admis à contrecœur au Parlement qu'il existait un «service secret» au sein de la GRC.»

9. Commission royale d'enquête sur la sécurité du Canada, Rapport, Centre d'édition du gouvernement du Canada, 1968, par. 297. 
Selon l'expression même de la commission McDonald, à partir de là, on a plutôt rebroussé chemin. À la suite des allusions publiques du Solliciteur général à l'opération $\mathrm{HAM}^{10}$, la GRC et son commissaire s'apprêtent à subir un véritable siège:

«En grande partie à la suite des évènements qui ont entraîné la création de la présente commission, le commissaire Simmonds a pris des mesures pour contrôler lui-même les pratiques et les politiques du service de sécurité" ".

Cette volonté d'autonomie est compréhensible si on tient compte de la nécessité, en démocratie, d'indépendance de la police face au pouvoir politique. Elle doit garder une certaine marge de manœuvre face aux forces politiques puisque l'application de la loi, en principe, ne souffre aucune exception. Une organisation policière est chargée de réprimer le crime. De sévir lorsqu'il y a action contraire à la loi. Le processus d'enquête est donc essentiellement réactif. C'est là que se situent les limites de l'intervention policière: elle entre en action lorsqu'un crime a déjà été commis ${ }^{12}$.

10. Cette année 1977 a été un véritable calvaire pour les services secrets. Pour un bon aperçu général, du point de vue de l'histoire du FLQ: Louis Fournier, F.L.Q., Histoire d'un mouvement clandestin, Montréal, Québec-Amérique, 1982, cinquième parti: L'après-Octobre. Au sujet de l'opération HAM, la source première est évidemment le minutieux rapport de Jean Keable et al., Rapport de la commission d'enquête sur des opérations policières en territoire québecois, Gouvernement du Québec, Ministère de la justice, 1981 et pour les amateurs d'une lecture plus "palpitante": Robert Dion, Les crimes de la Police montée, Montréal, Éditions coopératives Albert Saint-Martin, 1979, 233 p. avec photos et documents. Quoique ce dernier ouvrage soit superficiel, il résume efficacement, en quelques mots, la position du gouvernement: "Sachant le sujet délicat, le premier ministre Trudeau se porte à l'aide de son ministre pour tenter de justifier l'opération. Il commence tout bonnement par refuser de condamner la GRC d'être entrée illégalement dans les locaux d'une société commerciale montréalaise. Il suggère en outre que certains actes illégaux sont parfois nécessaires lorsque les intérêts de la sécurité nationale sont en jeu. Il ajoute même que dans de tels cas, les pouvoirs de la GRC devraient être plus étendus. Trudeau affirme en concluant que la GRC croyait que le PQ était engagé dans le terrorisme comme d'autres mouvements nationalistes." p. 97.

11. Commission d'enquête sur certaines activités de la Gendarmerie royale du Canada. Op. cit, p. 73.

12. Jean-Paul Brodeur, On Evaluating Threats to the National Security of Canada and the Civil Rights of Canadians, notes pour la conférence "Domestic Security: Issues for Democracty", octobre 1985, p. 3. 
Ce mécanisme a tendance à gripper lorsqu'il est question de renseignement de sécurité. Les limites de l'action policière acceptable sont difficiles à poser, déjà, lorsqu'il s'agit de démanteler des réseaux crapuleux clandestins, même lorsque leurs activités sont, sans aucune confusion, illégales. Délation, remise de peine, infiltration, invasion surprise de locaux font aussi partie de l'arsenal de la justice criminelle et posent un certain nombre de problèmes moraux qui ne peuvent être résolus par la seule application stricte de la loi. Le brouillard devient presque total dans le monde de la prévention du crime politique. L'appareil juridico-législatif ne suffit plus et laissée à elle-même, l'organisation de renseignement aura tendance à exagérer son rôle, multiplier les cibles, bref, voir partout des ennemis ${ }^{13}$.

Jusque dans les années 1970, les organisations de renseignement nord-américaines ont pu, relativement tranquilles, mener leurs petites activités au «service de la liberté». Mais de révélations en tempêtes médiatiques, le syndrome Watergate fait son œuvre et la classe politique questionne, tout à coup, ceux qui font «la guerre de l'ombre». L'opinion canadienne commence à se demander qui est au juste «THEE» que la Gendarmerie royale brandit dans sa devise.

À partir de l'été 1977, deux commissions d'enquête, à Ottawa et à Québec, s'arrachent témoins et documents secrets. De péripéties en croc-en-jambe, elles remettront des rapports à leurs gouvernements respectifs. Nous négligerons ces houleux épisodes pour voir plutôt ce qu'en ont été les résultats sur les conceptions et pratiques du renseignement au Canada. Le 28 juin 1984, la

13. Brodeur souligne, en plus de la tendance à ratisser large, que l'infiltration des groupes contestataires par les services secrets peut pousser ces groupes à des actes illégaux soit par la provocation pure et simple, soit en les empêchant de faire le choix de l'action légale. Attitude rationnelle du point de vue de l'agent infiltré: si le groupe n'est plus une menace, l'information ne vaut plus rien, il perd donc une source de revenu souvent importante. 
sanction de la Loi sur le Service canadien du renseignement de sécurité est la conséquence concrète de ces enquêtes.

Cette loi a modifié en partie le paysage du système de renseignement de sécurité: détachement du corps de la GRC et création d'un service civil (Service canadien de renseignement de sécurité, SCRS), encadrement des techniques de renseignement, établissement de mécanismes de contrôle interne (inspecteur général) et externe (Comité de surveillance des activités de renseignement de sécurité, CSARS). Ces nouvelles structures sont très jeunes. Il est un peu tôt pour dresser un constat sur le fonctionnement de ces organismes. Les services secrets en sont à s'habituer à leur nouveau costume. Ceci n'interdit pas, pour autant, de tenter une évaluation des difficultés qui se dressent face à la volonté de démocratisation de la pratique du renseignement. De la GRC au service civil, la transformation n'est pas facile et les facteurs de blocage sont nombreux. Des problèmes soulevés par le contenu même de la loi aux contraintes organisationnelles et administratives surgies de la manipulation du secret, on se demande s'il est possible de concevoir un service de renseignement vraiment audessus de tout soupçon.

\section{La loi: Sécurité et renseignement}

Assurer la sécurité de l'État, c'est en assurer l'intégrité et la pérennité. Là, déjà, commencent les difficultés. Qu'est-ce que l'État? Quel est son rapport au régime politique? Une volonté affirmée de changer le régime est-elle destructrice de l'État? Les réponses à ces questions ne peuvent être que normatives. Elles font appel à l'idée que se fait une collectivité du «bien commun", à la nature de ses objectifs, au choix des moyens pour y parvenir.

En 54 pages, la Loi sur le Service canadien de renseignement trace les bornes de ces choix de valeur. Entre les technicités, les renvois et les descriptions des nouveaux organismes, le centre 
nerveux de la question politique se retrouve dans la définition de la «menace envers la sécurité du Canada» qui tient en quatre paragraphes. Les deux premiers sont précis, à première vue:

«Constituent des menaces envers la sécurité du Canada les activités suivantes:

a) l'espionnage ou le sabotage visant le Canada ou préjudiciable à ses intérêts, ainsi que les activités tendant à favoriser ce genre d'espionnage ou de sabotage.

b) les activités influencées par l'étranger qui touchent le Canada ou s'y déroulent et sont préjudiciables à ses intérêts, et qui sont d'une nature clandestine ou trompeuses ou comportent des menaces envers quiconque ${ }^{14}$.

Ils concernent les activités d'espionnage et de sabotage en temps de paix ou de guerre. Dans le passé, la défense des intérêts nationaux a suscité, en temps de guerre, des abus, voire du racisme et de l'intolérance religieuse. Mais ces situations sont relativement exceptionnelles dans l'histoire. La protection de la souveraineté ne devrait pas faire problème en regard de la coutume et du droit tant intérieurs qu'internationaux ${ }^{15}$. Assurer la sécurité du Canada face à l'extérieur c'est comprendre le monde et la place qu'il y tient, identifier ses amis et ses ennemis, assurer sa position dans les conflits. Ce n'est pas la loi qui garantit que le service de sécurité y arrivera sans peine mais du moins, la mission est relativement claire. En temps de paix: prévoir la guerre, traiter des questions de violation clandestine de la souveraineté. En temps de guerre: combattre l'infiltration, se renseigner sur l'autre tout en le laissant le plus possible dans l'ignorance de nos propres intentions. Durant la Seconde guerre mondiale, le gouvernement canadien a choisi la méthode du bulldozer; un grand programme d'internement visant à priver les agents ennemis de tout contact

14. Loi sur le Service canadien de renseignement de sécurité, S.C., 1984, ch. 32, a. 2 .

15. Pour une discussion des problèmes d'évaluation de la menace extérieure: David Charters, Real or Perceived Theats to the Security of Canada: By What Criteria Should the Threat be Assessed, conférence "Domestic Security: Issues for Democracty", octobre 1985, photocopie (à paraître). 
intérieur. Beaucoup de groupes ont été touchés selon différents critères: race, origines, opinions politiques, opinions religieuses ${ }^{16}$.

Ainsi posée, la question paraît simple. Mais ce n'est qu'apparence. Depuis la Première guerre mondiale, le système international a subi des mutations qui ont transformé radicalement nos conceptions de la souveraineté. L'émergence de courants transnationaux, tant politico-idéologiques qu'économiques a ouvert des voies d'influence étrangère en marge des institutions étatiques. Prenant appui sur le développement des technologies de la communication, il est désormais possible, pour certains, de croire que l'on peut convaincre, recruter, embrigader les populations sans aucun égard pour la souveraineté. Dans un monde hétérogène où les idéologies s'affrontent, la frontière est ténue entre l'influence étrangère et les aspirations légitimes d'un peuple au changement. Durant les années 1930, il était tentant de ramener l'explosion de l'activité syndicale à un complot du communisme international ${ }^{17}$, au début des années 1980, Khaddafi était parfait dans son rôle de bouc-émissaire du terrorisme international. L'idée n'est pas neuve. À la fin du siècle dernier, une épidémie d'attentats anar-

16. «La GRC n'a guère pratiqué le contre-espionnage au cours de la guerre, sans doute en raison du vaste programme d'internement qui a peut-être privé les agents ennemis de contacts au Canada. Pendant les premières années de la guerre, avant que l'Union soviétique devienne notre alliée, l'activité anti-subversive portait surtout sur les présumées tentatives de communistes pour nuire à l'effort de guerre. La surveillance visait également le mouvement anti-conscriptionniste au Québec et les Témoins de Jéhovah. Ce dernier groupe, comme les communistes, avaient été déclaré illégal en vertu des Règlements concernant la défense du Canada. " Commission d'enquête sur certaines activités de la Gendarmerie royale du Canada. op. cit, p. 61.

17. "Entre les deux guerres, la GRC s'est surtout occupée [...] de la lutte contre la subversion. Ses principales cibles étaient les groupes communistes et les organisations ouvrières dirigées par les communistes. Les gouvernements successifs voulaient être mis au courant des projets révolutionnaires de ces organisations et de leurs appuis à l'étranger. $[\ldots]$

Dans les années 1930, la GRC avait infiltré à une très large échelle le mouvement communiste canadien." Commission d'enquête sur certaines activités de la Gendarmerie royale de Canada, op. cit, p. 59-60. 
chistes, en Europe, avait déjà fait croire à une grande conspiration mondiale patronnée par "certains États»... ${ }^{18}$ Devrions-nous mettre cette manie de tout expliquer par le complot d'un ennemi extérieur sur le compte de l'incompétence policière? Peut-être seulement en partie.

C'est ici que la notion à la mode de culture organisationnelle peut venir en aide. En effet, toute institution chargée de surveiller l'ordre public, que ce soit par le renseignement ou la répression, est profondément conservatrice. La GRC n'y échappe pas non plus ${ }^{19}$. Les individus plongés dans ce monde où ce qui est comme avant est ce qu'il y a de meilleur ne peuvent comprendre une volonté délibérée de changement. Ils ne peuvent admettre qu'un être humain qui se bat pour que les choses changent soit un humain normal ${ }^{20}$. La tentation est alors grande de jeter la faute sur ces étrangers qui ne sont pas comme nous. La sécurité extérieure sera donc liée étroitement aux perceptions de la menace intérieure.

Dans cette loi, le plus préoccupant est qu'elle touche la vie démocratique quotidienne, le fonctionnement dit normal de la société. Les paragraphes précédents situaient la menace chez l'ennemi, ou dans des activités servant les intérêts de cet ennemi au

18. "The high tide of terrorism in Western Europe was the anarchist "propaganda by the deed, in the 1890s. The exploits of Ravachol, Auguste Vaillant and Émile Henry between 1892 and 1894 created an enormous stir, and because the bomb throwing by individuals coincided with a turn in anarchist propaganda favouring violence, the impression of a giant international conspiracy was created, which in actual fact never existed. [...] The public at large was facinated by the secret and mysterious character of the anarchist groups; [...]. Governments and police forces who knew better saw no reason to correct this impression.»Walter Laqueur, The Age of Terrorism, London, George Weidenfeld \& Nicolson, 1987, p. 18.

19. «... la GRC renferme ses membres dans une éthique analogue à celle que l'on trouve dans les monastères ou les ordres religieux." Commission d'enquête sur certaines activités de la Gendarmerie royale du Canada. op cit, p. 104.

20. J'ai expliqué ailleurs les perceptions de normalité et de folie dans le discours officiel sur la criminalité politique: Janine Krieber, Le terrorisme et la responsabilité des intellectuels, Communication présentée à la conférence "Domestic Security: Issues for Democracty", Toronto, mai 1987. 
détriment de ceux du Canada. Les suivants sont plus étonnants:

«c) les activités qui touchent le Canada ou s'y déroulent et visent à favoriser l'usage de la violence grave ou de la menace de violence contre des personnes ou des biens dans le but d'atteindre un objectif politique au Canada ou dans un État étranger;

d) les activités qui, par des actions cachées et illicites, visent à saper le régime de gouvernement constitutionnellement établi ou dont le but immédiat ou ultime est sa destruction ou son renversement, par la violence» ${ }^{21}$.

Dans la définition la plus importante de cette loi, le gouvernement canadien a laissé toute une zone floue en faisant du concept de «menace envers la sécurité du Canada» une notion à double détente, une pièce à deux faces. La première face est classique: se défendre contre l'extérieur. La deuxième laisse perplexe. Étant donné les préoccupations du début des années 1980, on cherche vainement le mot terrorisme dans la loi. Le rapport McDonald en faisait mention en toute lettre. Le Législateur canadien, confronté aux difficultés de définition, a préféré évacuer cette notion en utilisant menaces envers la sécurité $d u$ Canada. Mais on retrouve dans la définition formelle de cette dernière "...l'usage de la violence grave ou de la menace de violence contre les personnes ou les biens dans le but d'atteindre un objectif politique...» ${ }^{22}$. Ceci ressemble étrangement à cette définition quasi-officielle du gouvernement américain: «... terrorism is the threat or use of violence for political goals» ${ }^{23}$.

La prochaine question, évidemment: qu'est-ce qu'un objectif politique? Les revendicateurs économiques dans un conflit, assistés sociaux, locataires, grévistes ont-ils des objectifs politiques? Les militants pour les droits sociaux des femmes, minorités, prisonniers,

21. Loi sur le service canadien de renseignement de sécurité.

22. Idem.

23. E. Nobles Lowe et D.H. Shargel, Legal and Other Aspects of Terrorism, New York, Practizing Law Institute, 1979, p. 94-95 cité par Alex P. Schmid, Political Terrorism: A Research Guide to Concepts, Theories, Data Bases and Litterature, SWIDOC et Transaction Books, Amsterdam, 1983, p. 147. 
malades? et d'autres qui défendent les droits des animaux de laboratoire... Aussi bien dire que tout est politique. En France, un groupe connu sous le nom de CLODO a fait sauter des ordinateurs pour protester contre l'invasion de la vie privée par la technologie informatique $^{24}$. En Allemagne, le SPK, groupe de malades contestant les méthodes psychiatriques conventionnelles, a été un noyau de recrutement de la Fraction armée rouge ${ }^{25}$. D'un certain point de vue, les objectifs de tout groupement contestataire peuvent être politiques.

De même pour la violence ou menace de violence. Des contrôleurs aériens font grève pour contester une nouvelle réglementation concernant la langue de travail - objectif politique. Des centaines de passagers sont prisonniers d'avions qui ne peuvent décoller. Viole-t-on leur droit fondamental à la liberté de déplacement? S'agit-il là d'une violence? La définition utilisée dans la loi peut donc, à la limite, justifier toute forme de cible. L'interprétation de ce qui constitue une menace à la sécurité de l'État est donc laissée aux bons soins du Service de sécurité.

Ce Service a été créé, entre autre, pour combattre «les activités qui, par des actions cachées et illicites, visent à saper le régime de gouvernement constitutionnellement établi au Canada». Quelles sont ces activités? La réponse, là aussi, est délicate. Tout dépend de la conception préalable de ce que sont les fondements d'un régime politique. Par exemple: les grèves dans les services publics. Les médias ne se gênent nullement pour affirmer haut que la population est prise en otage par des fonctionnaires trop gourmands. Point de vue qui se défend... Mais l'utilisation même du terme otage, connoté par l'idée de violence, rattache la grève,

24. Comité liquidant ou détournant les ordinateurs, voir Xavier Raufer, Terrorisme, Violence, Paris, J.J. Pauvert, 1984, p. 124-125.

25. Voir Wanda von Baeyer-Katte, "Das "Sozialistische Patientenkollectiv" in Heidelberg (SPK)», in Analysen zum Terrorismus, 3, Gruppenprozesse, Opladen, Westdeutscher Verlag, 1982: 183-316. 
en tant que moyen d'action politique, aux terroristes dont c'est la stratégie préférée. De là à concevoir les organisations porteuses de grèves - les syndicats - comme une menace à la stabilité du régime, le pas est vite franchi lorsque l'horizon des valeurs est réduit au maintien de la paix sociale à tout prix.

La loi tente d'éviter cet écueil en précisant que la surveillance ne doit viser que les activités «illicites»:

"La présente définition ne vise toutefois pas les activités licites de défense d'une cause, de protestation ou de manifestation d'un désaccord qui n'ont aucun lien avec les activités mentionnées aux alinéas a) à d) ${ }^{26}$.

Mais à la lumière des révélations au sujet du Service, dont le député néo-démocrate Svend Robinson s'est fait le critique implacable, la notion d'acte illicite, sans fondement légal très précis, semble assez large. Le fait est que cette idée dépend entièrement de la perception qu'aura l'organisation de renseignement de la menace, de son intensité et de son origine.

La perception de la menace: la question des cibles.

Le premier rapport de la commission McDonald insistait lourdement sur la présence de la menace, autant intérieure qu'extérieure:

«Il importe de se rappeler que les services de renseignements étrangers et les groupes terroristes menacent gravement la sécurité du Canada " ${ }^{27}$.

Il est permis de douter déjà que la menace soit si grave et importante, nous ne sommes ni à Belfast, ni à Beyrouth. Mais, forte de cette certitude, la commission ne donne pourtant pas les critères qui pourraient servir à l'identification et l'évaluation concrète

26. Loi sur le service canadien de renseignement de sécurité.

27. Commission d'enquête sur certaines activités de la Gendarmerie royale du Canada, Premier rapport, Sécurité et Information, Ottawa, Centre d'édition du gouvernement du Canada, 1979, p. 41. 
du risque ${ }^{28}$. Comme nous l'avons vu, même la loi, élaborée à partir du rapport, ne donne que des indications vagues qui, en dernière analyse, peuvent contenir à peu près tout. C'est donc dans son fonctionnement au jour le jour, de façon incroyablement autonome, que le Service fait cette évaluation, identifie des zones sensibles et, à l'intérieur de ces zones, choisit des cibles précises. Protégé par le secret, le Service prend des décisions politiques fondamentales en démocratie. Il décide, selon ses critères propres, ce qui est protestation légale et ce qui est «subversion». Il n'est guidé en cela que par un encadrement légal imprécis. Les caractéristiques de l'organisation en tant qu'administration quasi-policière et la spécificité technique de l'activité de renseignement deviennent donc les bases principales de l'évaluation de la menace.

Le Service canadien de renseignement et de sécurité est un clone de la Gendarmerie royale du Canada. Civil, il l'est devenu dans les textes mais peu dans la réalité. Au moment de sa formation, la majorité du personnel de l'ancien service a été tout simplement transférée et les modes de fonctionnement, recrutement et formation calqués sur ceux de la GRC ${ }^{29}$. À la première page de son rapport de 1987, le Comité de surveillance des activités de renseignement et de sécurité en fait encore un problème des plus urgents à régler:

«Nous craignons de plus en plus que la transformation en organisme civil n'avance trop lentement parce que le recrutement est fortement axé sur d'anciens policiers. Cela ne peut que perpétuer l'approche policière que le Parlement avait l'intention de changer en adoptant la Loi sur le SCRS ${ }^{30}$.

28. Jean-Paul Brodeur souligne, fort à propos, que la difficulté d'identification de la menace tient au fait qu'il faut prévoir un crime qui n'a pas encore été commis: op.cit.

29. Entretien informel avec Me Roger Tassé, Montréal le 23 septembre 1987. À cette période, Me Tassé a été chargé d'une enquête interne visant à restructurer le Service afin de lui donner une physionomie plus «civile».

30. Comité de surveillance des activités de renseignement et de sécurité, Rapport annuel, 1986-1987, Ottawa, Ministre des Approvisionnements et Services Canada, 1987, p. 1. 
La commission McDonald avait fait de la transformation du service de renseignement en service civil une condition fondamentale de démocratisation, un pas essentiel vers la transparence. Un examen minutieux des activités de la GRC a dicté à la commission des conclusions très dures. Actes illégaux, violation de la vie privée, exagérations de toutes sortes, les Tuniques rouges déguisées en complet-veston pour l'occasion - s'étaient taillé une zone d'activités secrètes sur laquelle la Commission Keable a projeté une lumière crue $^{31}$.

La création d'un organisme autonome s'est révélée fort loin de la panacée attendue par les différentes commissions. Le recrutement et la formation semblent être un problème de base ${ }^{32}$. L'analyse du renseignement exige de grandes capacités intellectuelles: vaste connaissance du monde politique, esprit de synthèse exceptionnel, humilité devant les faits... Il est permis de douter que la formation donnée aux policiers (ou ex-policiers) canadiens, beaucoup plus axée sur l'action que sur l'abstraction, les aide à développer ces aptitudes. Incapables d'interpréter les données sociopolitiques qui sont carrément sous leurs yeux, ils préféreront monter des «coups». Un renseignement venu d'un informateur secret ou issu d'un vol de documents gagne, par son mystère, une vérité intrinsèque. L'opération clandestine, surtout pour l'évaluation du risque de violence dans l'opposition politique, est la solution de facilité. Une analyse, même excellente, doit être justifiée pied à pied. Elle demande une expertise politicosociologique au-dessus de la moyenne et les services de renseignement se méfient de ces sciences qui produisent des «révolutionnaires». Il y a donc peu, ou pas, de personnel capable de recourir à

31. Nous ne revenons pas en détail sur ces révélations. Nous renvoyons le lecteur au rapport de la commission Keable.

32. C'est avec raison que le rapport Osbaldeston op.cit fait du recrutement et de la formation l'élément central de la réforme du service de renseignement. 
l'argument serré, sans faille, que demande l'analyse des sources ouvertes. On s'entraîne peu à cet exercice, dans la police, et on se méfie des jeunes intellectuels qui, pour leur part, ne semblent pas très attirés par cette carrière ${ }^{33}$.

La plupart des enquêteurs qui ont examiné les activités de renseignement en Occident en sont arrivés à cette conclusion. Ces services ont tendance à surestimer la menace et à aecorder leurs opérations clandestines à cette vision du monde. Ils ont donc proposé des solutions voisines: plus de contrôle. Bref, surveiller les espions ${ }^{34}$.

\section{Une solution: la responsabilité politique}

En toute logique, la responsabilité politique dans ce domaine est une garantie que toutes les sociétés démocratiques ont le devoir de se donner. Le rapport de la commission Mackenzie allait en ce sens et n'a pas été suivi des décisions nécessaires. La commission $\mathrm{McDonald}$ est donc revenue en force sur ce point:

«En premier lieu, le gouvernement responsable suppose, selon nous, la mise au point d'un dispositif en vertu duquel les responsables d'enquêtes et d'autres mesures de sécurité sont comptables aux ministres de la couronne, lesquels doivent rendre compte au Parlement ${ }^{35}$.

Proposition vertueuse s'il en est. Mais de la recommandation au fait, le chemin est long et rempli d'embûches. La responsabilité n'est pas un rempart sans faille. On a vu, dans l'histoire de l'espionnage, des responsables politiques, et non les moindres,

33. Actuellement, le SCRS ferait un effort de diversification du recrutement mais il semble manquer encore beaucoup de francophones, de femmes et de diplômés universitaires.

34. U.S. Senate Report no. 94-755, Foreign and Military intelligence. Select Comittee to Study Governmental Operations, 94th Cong., 2d sess., 1976. Australia, Fourth Report of The Royal Commission on Security and Intelligence, Canberra, Government Printer, 1978. Il y a eu aussi différents rapports en Nouvelle-Zélande et au Royaume-Uni.

35. Commission d'enquête sur certaines activités de la Gendarmerie royale du Canada. op. cit, p. 45. 
identifier l'intérêt national à celui de leur parti. Ceci dit, les difficultés d'exercice d'une responsabilité politique efficace tiennent au désintérêt relatif du public face à ces questions; indifférence dûe, en grande partie, à la complexité et l'étendue de la communauté du renseignement mais aussi à la manie du secret et de la duplicité.

L'espion réel est beaucoup plus près de Smiley que de James Bond. Il faut le génie d'un Le Carré pour passionner les foules. Du génie ou des révélations exceptionnelles. Aux États-Unis, comme au Canada, la volonté du politique de contrôler les services secrets s'est manifestée à la suite d'un scandale. Fin décembre 1974 , la poussière du Watergate n'est pas encore retombée et, dans le sillage du scandale du siècle, le grand journalisme d'enquête s'excite encore sur la CIA et le FBI. Alors paraît dans le New York Times une série d'articles signés par Seymour M. Hersh. Une bombe dans les milieux du renseignement. L'amorce se lisait comme ceci:

"The Central Intelligence Agency, directly violating its charter, conducted a massive, illegal domestic intelligence operation during the Nixon administration against the antiwar movement and other dissident groups in United States, according to well-places Government sources.

An extensive investigation by The New York Times has established that intelligence files on at least 10,000 American citizens were maintained by a special unit of the C.I.A. ... ${ }^{36}$

La suite d'articles révèlera, jour après jour, un programme d'infiltration massive des campus universitaires - seulement à Columbia, plus d'une vingtaine d'espions se sont mêlés à la protestation pacifiste. Infiltration, ouverture du courrier, écoute téléphonique, études psychologiques, plans de discrédit visant des leaders contestataires, le programme était ambitieux et dépendait

36. S.M. Hersh, "Huge C.I.A. Operation Reported in U.S. Against Antiwar Forces, Other Dissidents in Nixon Years", The New York Times, Sunday, December 22, 1974 , p. 1, section 1 . 
exclusivement de Richard Helms, directeur de 1966 à $1973^{37}$. Il s'est avéré qu'il s'agissait là d'un coulage volontaire venant d'une faction de la CIA. Les documents, concernant l'opération CHAOS, avaient été tirés d'une enquête interne menée par le successeur de Helms, James Schlesinger, en mai $1973^{38}$. L'amendement Hughes-Ryan au Foreign Assistance Act, qui concernait plus particulièrement l'aide militaire en Angola, donne au Congrès un droit de regard sur les activités secrètes de renseignement et deux comités sont formés. Ils deviendront célèbres sous les noms de leurs présidents, Frank Church et Otis Pike ${ }^{39}$. Dès le début, les deux comités ont adopté un style agressif d'enquête qui s'est un peu atténué lors de la création des comités permanents de surveillance (Select Commitees on Intelligence). À partir de ce moment, le système américain intrégrait un processus permanent de surveillance des activités de renseignement. Contrairement aux positions des commissions d'enquête ad hoc toujours réunies à la

37. Les documents déclassifiés sous le Freedom of Information Act sont recensés périodiquement sous forme d' "Abstract». Une consultation au sujet de l'opération CHAOS et une vérification partielle du contenu des microfiches à la Bibliothèque du Congrès à Washington m'a convaincue qu'il s'agissait en très grande partie de rapports d'agents infiltrés dans les milieux étudiants. La Commission McDonald a aussi remarqué la méfiance presque atavique de nos services envers les étudiants: " $\grave{A}$ la lumière de ces faits, nous en sommes venus à la conclusion, vers la fin des années 1960, que la GRC a entrepris, sans l'approbation du gouvernement, un important programme visant à multiplier et à améliorer les contacts avec les professeurs d'université. Ce programme a été instauré à cause du militantisme qui était en progression dans les universités et du terrorisme qui se développait dans quelques-unes. " p. 362.

38. Dans une tentative pour rassembler les données sur les actions de la CIA non conformes à sa charte, le directeur s'est vu bientôt inondé: 693 cas de dépassement possible des attributions se sont retrouvés sur son bureau. Loch K. Johnson, A season of Inquiry; The Senate Intelligence Investigation, Lexington, The University Press of Kentuky, 1985 , p. 10.

39. Pour une relation détaillée des enquêtes menées par le Comité du sénat présidé par Frank Church: ibid 317p. L'amendement devient loi le 30 décembre 1974: "This statute, which require the president to approve and report to congress all important covert actions, represented the first succesfull effort by legislators to place controls over the CIA since its creation" idem, p. 10. 
suite de scandales publics, la surveillance permanente se base sur une acceptation du principe même du renseignement plutôt que sur sa contestation. Les objectifs-ne sont plus de dénoncer les activités secrètes pour les interdire mais de les tenir dans des limites moralement et légalement acceptables ${ }^{40}$.

C'est dans l'esprit d'une surveillance constante qui évite la confrontation que, depuis l'adoption de la Loi sur le Service canadien de sécurité, le gouvernement canadien surveille ses espions. Tout en constituant un service distinct chargé du renseignement de sécurité, la loi le flanque d'un "chien de garde»: le Comité de surveillance des activités de renseignement de sécurité. Comme les comités du Congrès américain, la commission McDonald a assuré son héritage. Elle avait proposé un processus de révision sous la responsabilité du Sénat et de la Chambre, la loi a opté pour un comité de révision indépendant. Le Comité sera formé du président et de deux à quatre membres qui ne font partie ni du Sénat, ni de la Chambre des Communes; assurance partielle contre la mainmise des partis sur les services secrets. Ces nominations, d'une durée de cinq ans, renouvelables une seule fois, sont la responsabilité du Gouverneur général en conseil après consultation du premier ministre, du chef de l'opposition et du chef de chacun des partis qui sont représentés par au moins douze députés. Le comité actuel est donc un compromis entre le modèle américain qui reproduit à peu près la composition des assemblées et un mode de contrôle purement administratif.

Le comité est tenu, chaque année, de présenter un rapport dont une version épurée, pour raison de sécurité, est rendue publique. Une des vertus de ces rapports est, entre autre, de donner périodiquement aux médias l'occasion de s'intéresser aux

40. Gary J. Schmits «Congressional Oversight: Form and substance», in Gerald W. Hopple, Bruce W. Watson, The military Intelligence Community. Boulder Col., Westview Press, 1986: 265-288. 
services de renseignement, en dehors du sensationnalisme, en faisant aux journalistes cadeau d'informations pré-digérées.

Le choix entre les deux grandes méthodes de renseignement, l'une ouverte, l'autre secrète, génére un conflit entre le Service de renseignement et le Comité de surveillance. Ce dernier, à la suite de la Commission McDonald, affirme bien haut sa préférence pour les sources ouvertes tandis que le Service - fidèle à la tradition de la GRC — semble être plus à l'aise dans la préparation d'opérations clandestines:

"... nous croyons fermement que le SCRS n'a pas suffisamment recours aux sources ouvertes comme solution de rechange aux enquêtes clandestines ${ }^{41}$.

Au-delà de la facilité, cette attitude peut être difficilement expliquée. Il n'y a peut-être que lourdeur historique, ce que nous avons déjà appelé culture organisationnelle et qu'on pourrait qualifier ici de manie. L'espion sur le terrain, l'indicateur infiltré ont pour seul métier le mensonge. Solidarité à l'intérieur et méfiance tout azimut, même face aux pouvoirs publics qui devraient contrôler leurs activités, les services secrets sont passés maîtres dans l'art de la dissimulation.

Le sommet de l'incompétence serait évidemment, pour ces services, d'être infiltrés par des journalistes. Véritable créateur de l'opinion, le rouage médiatique est pourtant essentiel dans le processus de contrôle. Mais fouiller le monde du renseignement, pour en dénoncer les abus, demande une absence totale de préjugés, de bonnes qualités d'enquêteur, de l'abnégation et de la constance. C'est dans les difficultés quotidiennes du travail du journaliste que s'articulent leurs problèmes. Les contraintes de temps et d'espace font que les médias répercutent beaucoup plus qu'ils n'analysent. Le monde du renseignement est complexe et morcelé, difficile à pénétrer. Autour du Service lui-même, c'est une véritable

41. Comité de surveillance des activités de renseignement et de sécurité, op. cit. p. 1 . 
communauté, disséminée et solidaire qui, et pour cause, s'ouvre peu au regard des gens curieux ${ }^{42}$. Cette manie de la protection contre la plus petite fuite s'exerce non seulement face à l'étranger, ce qui va de soi, mais aussi vis-à-vis des hommes politiques dont on ne sait jamais ce qu'ils peuvent révéler aux journalistes.

«La preuve a été faite que des ministres fédéraux de la Couronne responsables de la GRC ont été trompés par celle-ci et qu'à d'autres occasions des renseignements pertinents ou importants ont été délibérément dissimulés » ${ }^{43}$.

\section{Les espions savent bien cacher les choses, surtout ce qui se} passe chez eux. Tous ceux dont le travail est de pénétrer un peu le mystère s'en sont plaint un jour ou l'autre:

"Even if the overseers had wanted to know, they might have failed to obtain the full story. Former CIA Director Allen W. Dulles (1953-61) told the Warren Commission that when he was at the agency's helm, he felt obliged to tell the truth only to one person; the president ${ }^{44}$.

La réticence des services secrets à dévoiler leurs pratiques puise à deux sources. La première est basée sur une interprétation large du principe de «dénégation plausible»: ministres ou chefs d'État, les responsables politiques ne doivent jamais être mis en situation de commenter publiquement des opérations secrètes. La

42. De plus, lorsqu'un coin du voile est levé, on reste béat. L'ascension d'un document ou d'une information vers le secret cosmique ne semble dépendre ni de son contenu, ni de son origine, mais d'un certain nombre de manipulations bureaucratiques. Il y a deux façons de désinformer: tout cacher ou noyer l'interlocuteur sous une masse d'informations non pertinentes. Le gouvernement canadien semble utiliser un peu des deux. Allié au fait que les espions doivent aussi justifier leur salaire en produisant quelque chose, le résultat est qu'on ratisse plus large que nécessaire.

$\mathrm{J}$-P. Brodeur raconte à ce sujet une anecdote significative. Un informateur arrive à l'université, une bobine précieuse sous le bras. Ultra secret. Au visionnement, le professeur se rend compte que le film avait été diffusé auparavant à la télévision d'État. Dans ce cas, ce qui est un secret, c'est que ce film soit classé secret...

43. Commission d'enquête sur certaines activités de la Gendarmerie royale du Canada. op. cit, p. 103 et un peu plus loin, le rapport ajoute: «... ces comportements sont bien établis et découlent d'une attitude fort répandue dans la Gendarmerie, à savoir qu'elle n'a pas à répondre de ses actes à l'autorité civile.» p. 104.

44. Loch K. Johnson, op. cit, p. 6. 
solution la plus simple est donc de les laisser dans la plus parfaite ignorance. Ils ont tendance, d'ailleurs, à accepter assez facilement cette situation estimant, probablement, qu'ils ne peuvent être tenus responsables de ce qui se passe dans leur $\operatorname{dos}^{45}$. La seconde est une préoccupation - fort légitime en soi - de l'image publique mais qui s'exprime ici dans l'idée que ce qui reste caché ne peut nuire à l'organisation. Comme la tâche principale d'un service de renseignement est de manipuler le secret d'État, il n'est pas étonnant que ses chefs et ses membres se servent de leur expertise, beaucoup mieux que dans d'autres organismes, pour se protéger dans les cas d'activités douteuses, à la limite de la légalité.

«Le trait commun de tous les incidents est la disposition des membres de la GRC à tromper ceux qui, en dehors de la Gendarmerie, exercent une autorité ou une compétence constitutionnelle sur eux ou sur leurs activités » ${ }^{46}$.

Le Comité de surveillance avait donc un long chemin à parcourir. La lecture des rapports annuels donne l'impression que le Service s'ouvre assez bien à l'organisme de surveillance. Mais au détour de quelques phrases, on sent un malaise. Ainsi, le rapport de 1986 révèle à mots couverts que les relations ne sont pas toujours au beau fixe:

«Nos relations avec le SCRS s'améliorent, et nous sommes encouragés par les efforts consciencieux que font certaines personnes au sein du Service afin d'établir un climat de respect mutuel et de collaboration dans l'exercice de nos mandats respectifs. On semble toutefois persister à croire au sein du Service que le Comité et l'inspecteur général constituent une présence quelque peu gênante... " ${ }^{47}$

45. Ce fut l'attitude du premier ministre Trudeau au sujet de l'opération HAM. Voir note 6 supra.

46. Commission d'enquête sur certaines activités de la Gendarmerie royale du Canada. op. cit, p. 103.

47. Comité de surveillance des activités de renseignement et de sécurité, Rapport annuel, 1985-1986, Ottawa, Ministre des approvisionnements et services Canada, 1987, p. 11. 


\section{Conclusion}

Notre projet était de voir à quels blocages se heurtait une volonté de démocratie et de transparence face à la nécessité d'entretenir un système de renseignement de sécurité. Nous en arrivons à la conclusion que beaucoup de ces difficultés sont d'ordre structurel. Certaines tiennent aux ambiguïtés juridiques dans la définition du crime politique, lorsque notre régime est précisément fondé sur l'évacuation de cette notion. Penser et discuter publiquement de l'utilisation politique de la violence n'est pas un crime au Canada. Beaucoup d'intellectuels ont fait de la nécessité historique de la révolution le centre de leurs analyses, et révolution implique guerre révolutionnaire. Passer aux actes, entrer dans la clandestinité, est évidemment autre chose. Mais de tous ceux qui en parlent, lesquels agiront?

C'est ici que se dresse le mur auquel se heurtera longtemps tout service de renseignement. C'est une organisation de prévention: sa tâche est de prédire des comportements socio-politiques - et nous savons tous l'entreprise franchement ardue. En pure abstraction logique, tout mécontentement social, politique ou économique peut en arriver à générer et justifier la violence; il suffit d'en rassembler les conditions spécifiques. Quelles sont-elles? Les recherches donnent peu d'indications, ou trop. Les solutions sont encore loin.

Pour le moment, le gouvernement canadien a choisi la voie d'une stricte légalité. Les techniques utilisées par les services de renseignement sont contrôlées de près et le Comité de surveillance s'est attaqué à un problème de taille: la mentalité policière. Le nouveau service ne réussira évidemment pas à se débarasser du jour au lendemain du lourd héritage laissé par la GRC mais il semble que les membres et le personnel du Comité se rendent compte de l'ampleur du travail et surtout qu'une gestion gouvernementale responsable du secret d'État passe par la qualité et l'indépendance de leurs enquêtes sur les espions. 